\title{
Structural features of the formation of iHOF materials
}

\author{
Petra Bombicz ${ }^{a}$, Laura Bereczkia ${ }^{\text {, Nóra Veronika Maya, }}$ \\ Roberta Palkó ${ }^{b}$, Dániel Vajk Horváth ${ }^{b}$, Tibor Soós ${ }^{b}$, Tamás Holczbauera,b \\ ${ }^{a}$ Research Laboratory of Chemical Crystallography, \\ bInstitute of Organic Chemistry, \\ of Research Centre for Natural Sciences, Hungarian Academy of Sciences \\ bombicz.petra@ttk.mta.hu
}

Solid structures with large volume areas have a broad range of applications, such as sensing, drug delivery, heterogeneous catalysis, separation, storage, etc. They are chemically diverse materials, and their macroscopic properties can be tailored. Porous hydrogen-bonded organic frameworks (HOFs) are in the forefront of interest in the recent years. The long-range periodicity in crystals of HOFs is a product of the directionally specific short-range intermolecular interactions. In order to be able to control the self-assembly of HOFs, it is important to understand molecular recognition principles. Most of the organic frameworks described are electronically neutral. Anyhow, a useful method to increase the strength of the hydrogen bond is to incorporate charged sites to assist hydrogen bonding of the building blocks. Ionic hydrogen-bonded organic frameworks (iHOFs) is a new area of research. We investigate the conditions of iHOF formation:

a., the intramolecular interactions, responsible for the inflexibility of the molecule, b., the intermolecular interactions, responsible for framework construction, c., the terminal spacer groups for void formation,

d., the molecular symmetries, important in the tightening of the molecules, and e., all the afore mentioned features affect the crystal symmetry which may coincide with the molecular symmetry.

We study the electrostatic and steric properties of our framework constructing compound family by systematic chemical alterations. It results also in polymorphic and solvatomorphic structures. Our study may support the still challenging design and preparation of framework structures with high porosity, and describes the new type of iHOF structures.

This work was supported by the National Research, Development and Innovation Office-NKFIH through OTKA K115762, K116150, K124544 and KH129588; and by the Bolyai Research Scholarship of HAS (TH).

\section{Reference}

[1] Horváth D.V., Holczbauer T., Bereczki L., Palkó R., May N.V., Soós T., Bombicz P. (2018)

CrystEngComm, 20, 1779-1782. 